\title{
Valeur alimentaire des marcs de raisin \\ II - Effet d'un traitement à la soude sur la valeur alimentaire chez le mouton de marc de raisin épuisé à la vapeur et ensilé
}

\author{
A. LARWENCE, Fatma HAMMOUDA et Yamina GAOUAS \\ avec la collaboration technique de Samia ABAdA, Rachida BENAIK, Nacera Ouchat \\ Institut national agronomique, Département de Zootechnie \\ El Harrach, Alger
}

\begin{abstract}
Résumé
La digestibilité des marcs de raisin (distribués ad libitum) traités ou non à la soude à la dose de 0,67 p. 100 ou de 1,11 p. 100 de la matière sèche a été étudiée sur des lots de 4 moutons.

Il ressort que le traitement à la soude fait passer leur digestibilité de 32 p. 100 (marcs non traités) à 44 p. 100 (marcs traités à 0,67 p. 100); pour la dose de 1,11 p. 100, nous enregistrons une légère diminution de la digestibilité de la matière organique qui se situe alors à 41 p. 100 .

L'amélioration de la digestibilité porte sur tous les composants des marcs et ceci particulièrement sur la cellulose Van Soest et sur les lipides dont les digestibilités passent respectivement de 25 à 61 p. 100 et de 46 à 69 p. 100. La digestibilité de l'azote reste en moyenne faible $(20$ p. 100$)$ mais varie de 10 (marcs non traités) à 29 p. 100 (marcs traités à 0,67 p. 100$)$.

L'ingestibilité du produit est particulièrement importante $(124 \mathrm{~g}$ en moyenne $/ \mathrm{kg}$ $\left.\mathrm{P}^{0,75}\right)$; elle n'a pas varié sous l'action du traitement à la soude malgré une dépréciation de la qualité de l'ensilage (l'acide butyrique passe de $1,4 \mathrm{~g}$ à $44 \mathrm{~g} / \mathrm{kg}$ de matière sèche lorsque la dose de soude passe de 0 à 1,11 p. 100).

Cette forte ingestibilité, alliée à l'amélioration de la digestibilité de la matière organique confère aux marcs de raisin traités à la soude, une valeur alimentaire couvrant 1,5 fois les besoins d'entretien alors que non traités, la couverture ne représente que 55 p. 100.

Les marcs de raisin traités à la soude constituent donc un aliment intéressant pour des moutons à l'entretien ou à croissance modérée.
\end{abstract}

\section{Introduction}

Les mares de raisin, avec une production mondiale annuelle de plus de 7 millions de tonnes, constituent l'un des plus importants sous-produits agricoles après les pailles de céréales ( 3 milliards de tonnes selon JACKsON, 1977). 
Leur faible valeur nutritive (digestibilité de la matière organique comprise entre 24 et 40 p. 100) est rapportée par de nombreux auteurs : MAzziotri et al. (1970), Economides \& Hadidemetriou (1974), Reyne \& Garambois (1977), Dumont \& TisSERAND (1978), Hammouda \& LARWENCE (1981).

La digestibilité de la matière organique est inférieure à celle de la paille de blé qui selon Harton (1978) est de l'ordre de 45 p. 100 ; mais leur forte ingestibilité (environ $2 \mathrm{~kg}$ de matière sèche par mouton et par jour), leur confère une valcur alimentaire comparable à celle observée pour les pailles de céréale. Elle devient supérieure lorsquils sont complémentés en énergie et en azote : dans ce cas, la nature de la source azotée de complémentation est importante (plus particulièrement le degré de fermentescibilité de son azote). C'est ainsi que Larwence \& Yahiaour (1983) sur les 8 sources azotées de complémentation testées observent les meilleurs résultats avec le tourteau de colza.

Depuis quelques années, le traitement à la soude est préconisé par de nombreux auteurs comme un moyen satisfaisant pour améliorer la valeur alimentaire des fourrages riches en glucides pariétaux (Revue de JACKSON, 1977). La soude, en solubilisant en partie la cellulose, les hémicelluloses et la lignine, augmente la digestibilité de ces fourrages.

Compte tenu de la teneur élevée en glucides pariétaux des marcs de raisin (72 p. 100 selon Hammouda, 1980), lapplication d'un traitement à la soude, est susceptible, à l'image des résultats obtenus avec les pailles de céréale, d'améliorer l'utilisation digestive de ce sous-produit de la vinification.

\section{Matériel et méthode}

\section{A. Digestibilité in vivo, ingestibilité et appréciation de la qualité de l'ensilage}

La méthode de détermination de la digestibilité in vivo, les analyses chimiques, la préparation des ensilages ainsi que lappréciation du faciès fermentaire ( $\mathrm{pH}$, azote soluble, acides gras volatils et ammoniac) ont été décrites par LARwence \& YahiaouI (1983). Les constituants pariétaux sont déterminés par la méthode de VAN SoEST (1967).

Pour ce qui concerne l'ingestibilité, elle est mesurée durant une période de 4 semaines sur 6 moutons de race Ouled-Djellal pesant entre 48 et $51 \mathrm{~kg}$ accoutumés pendant 2 semaines aux régimes et maintenus en cage individuelle. Ils reçoivent à volonté, les rations distribuées deux fois par jour ( 8 heures et 17 heures).

\section{B. Traitement des marcs de raisin}

Tenant compte de la forte ingestibilité du produit, il convient d'utiliser des faibles doses de soude pour éviter une ingestion trop élevée de cette substance par les animaux.

Nous avons employé trois niveaux de soude : 30, 50 et $70 \mathrm{~g}$ par litre d'eau. Ce volume est utilisé pour traiter $10 \mathrm{~kg}$ de marc à $45 \mathrm{p} .100$ de matière sèche, soit respectivement $0,67,1,11$ et 1,56 p. 100 ou $1,48,2,46$ et 3,44 p. 100 selon que la soude est exprimée en fonction de la matière sèche ou par rapport au produit frais. 
Les marcs, par lots de $170 \mathrm{~kg}$ sont étalés sur un film plastique et arrosés avec 17 litres de la solution de soude à l'aide d'un arrosoir de jardin. Après un malaxage vigoureux avec des pelles et les pieds, le produit est ensilé dans des fûts de 200 litres munis de trous pour permettre l'évacuation du jus. Le désilage est effectué 3 mois plus tard.

\section{Digestibilité in vitro}

Pour choisir les doses de traitement sur lesquelles portent la digetibilité in vivo, nous effectuons in vitro un test de disparition de la matière sèche selon Tilley \& Terry (1963). Les animaux donneurs de jus reçoivent par jour : $1 \mathrm{~kg}$ de foin de vesce-avoine de qualité moyenne et $200 \mathrm{~g}$ de concentré «ovin» du commerce.

\section{Calcul}

La matière sèche des ensilages est corrigée par l"équation de ScHoch (1949).

La valeur énergétique des rations est exprimée en unité fourragère lait.

Le rendement $\mathrm{Kl}$ est estimé à 60 p. 100 et la digestibilité de l'énergie par l'expression : $\mathrm{dE}=1,0087 . \mathrm{dMO}-0,0377$ (I.N.R.A., 1978).

Les résultats, font l'objet de comparaison de variance (test de Snedecor) avant d'éventuel comparaison de moyenne (test de Student).

\section{Résultats}

\section{A. Composition chimique}

Les résultats consignés dans le tableau 1 sont comparables pour ce qui concerne les marcs non traités (MNT) à ceux rapportés par nous-mêmes dans le précédent article (LARWENCE \& Yahiaoui, 1983). Traités, certains composants des mares de raisin sont modifiés : la teneur en matières azotées, diminue par suite d'unc perte d'azote dans le jus d'ensilage; les matières minérales augmentent avec la dose de soude ce qui est normal. Les teneurs en cellulose brute, en neutral detergent fiber (NDF), et en acid detergent fiber (ADF) évoluent sous l'effet du traitement et ceci, particulièrement pour celle en NDF.

Par contre, nous observons une diminution de 2 points de la tencur en hémicellulose et de 17 points pour la teneur en acid detergent lignin (ADL).

\section{B. Bilan des silos}

Les pertes totales par pourriture sélèvent à 5,1 p. 100 pour MNT, 3,1 p. 100 pour les marcs traités à 0,67 p. 100 de soude (MT 0,67 ) et à 1,6 p. 100 pour les marcs traités à 1,11 p. 100 de soude (MT 1,11). La soude aurait donc un rôle de protection de l'ensilage contre la pourriture de surface.

Les paramètres fermentaires sont rapportés dans le tableau 2. Le traitement fait augmenter légèrement la quantité d'ammoniac et d'azote soluble mais dans l'ensemble, l'azote des marcs de raisin demeure pratiquement insoluble puisque la somme de ces deux formes d'azote n'atteint pas 2 p. 100 de l'azote total. 


\section{TABleau 1}

Composition chimique des aliments (\% MS).

Chemical composition of feed (\% dry matter).

\begin{tabular}{|c|c|c|c|}
\hline & $\begin{array}{c}\text { Marc non traité } \\
\text { Untreated grape } \\
\text { marc }\end{array}$ & $\begin{array}{c}\text { Marc traité } \\
0,67 \% \\
\text { Treated } 0.67 \% \\
\end{array}$ & $\begin{array}{c}\text { Marc traité } \\
1,11 \% \\
\text { Treated } 1.11 \% \\
\end{array}$ \\
\hline $\begin{array}{c}\text { Matière sèche corrigée }- \text { Corrected dry } \\
\text { matter } \ldots \ldots \ldots \ldots \ldots \ldots \ldots \ldots \ldots \ldots \ldots\end{array}$ & 44,72 & 43,38 & 42,76 \\
\hline Matières azotées - Crude protein & 14,43 & 13,85 & 13,30 \\
\hline Matière grasse - Fat $\ldots \ldots \ldots \ldots \ldots$ & 7,81 & 8,19 & 7,80 \\
\hline Cellulose Weende - Cellulose Weende .. & 27,01 & 29,93 & 31,31 \\
\hline Matière minérale - $A s h$ & 5,31 & 6,57 & 6,91 \\
\hline Neutral detergent fibre (NDF) & 74,15 & 84,96 & 88,13 \\
\hline Acid detergent fibre (ADL) & 59,44 & 71,68 & 75,98 \\
\hline Acid detergent lignin $(A D L)$ & 40,34 & 30,81 & 23,01 \\
\hline
\end{tabular}

Note : Il semble que le traitement à la soude a insolubilisé une partie des constituants cytoplasmiques puisque pour MT 0,67 et MT I, II, la somme MAT + MG + cendres + NDF est respectivement de 114 et $116 \mathrm{p}$. 100 .

\section{TABLEAU 2}

Caractéristiques fermentaires de l'ensilage de marc de raisin traité ou non à la soude. Fermentation characteristics of grape marc silage untreated or treated with sodium hydroxide.

\begin{tabular}{|c|c|c|c|}
\hline & $\begin{array}{l}\text { Marc non traité } \\
\text { Untreated grape } \\
\text { marc }\end{array}$ & $\begin{array}{c}\text { Marc traité } \\
0,67 \% \\
\text { Treated } \\
\text { grape marc } \\
0.67 \% \\
\end{array}$ & $\begin{array}{c}\text { Marc traité } \\
1,11 \% \\
\text { Treated } \\
\text { grape marc } \\
1.11 \%\end{array}$ \\
\hline $\begin{array}{l}\text { Matière sèche non corrigée } \ldots \ldots \ldots \ldots \\
\text { Non-corrected dry matter }\end{array}$ & 43,01 & 41,26 & 39,74 \\
\hline 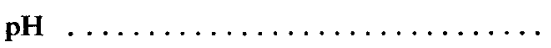 & 3,37 & 4,83 & 5,47 \\
\hline $\begin{array}{l}\text { Azote ammoniacal }(\% \mathrm{~N} \text { total }) \ldots \ldots \ldots \\
\text { Ammonia nitrogen }(\% \text { total nitrogen })\end{array}$ & 1,52 & 2,11 & 2,88 \\
\hline $\begin{array}{l}\text { Azote soluble }(\% \text { N total) } \ldots \ldots \ldots \ldots \\
\text { Soluble nitrogen (\% total nitrogen) }\end{array}$ & 1,03 & 1,33 & 1,55 \\
\hline $\begin{array}{l}\text { Acide acétique }(\mathrm{g} / \mathrm{kg} \text { MS }) \ldots \ldots \ldots \ldots \\
\text { Acetic acid }(g / \mathrm{kg} \text { dry matter })\end{array}$ & 19,6 & 49,3 & 78,9 \\
\hline $\begin{array}{l}\text { Acide butyrique (g/kg MS) } \ldots \ldots \ldots \ldots \\
\text { Butyric acid }(g / \mathrm{kg} \text { dry matter })\end{array}$ & 1,4 & 13,9 & 44,2 \\
\hline $\begin{array}{l}\text { Acide lactique ( }(\mathrm{g} / \mathrm{kg} \text { MS }) \ldots \ldots \ldots \ldots \\
\text { Lactic acid }(g / k g \text { dry matter })\end{array}$ & 8,6 & 6,1 & 2,2 \\
\hline
\end{tabular}


Par contre, les teneurs en acide acétique $\left(C_{2}\right)$ et en acide butyrique $\left(C_{4}\right)$ augmentent dans de fortes proportions. $C_{2}$ passe de $19,6 \mathrm{~g} / \mathrm{kg}$ de matière sèche pour MNT à 49,3 et $78,9 \mathrm{~g}$ respectivement pour $\mathrm{MT} 0,67$ et $\mathrm{MT} 1,11$. L'augmentation est encore plus spectaculaire pour $\mathrm{C}^{4}$ : de 1,4 à 13,9 et $44,2 \mathrm{~g} / \mathrm{kg}$ de matière sèche respectivement.

La teneur en acide lactique varie en sens inverse : elle se situe à $8,6,6,1$ et $2,2 \mathrm{~g} / \mathrm{kg}$ de matière sèche respectivement pour MNT, MT 0,67 et MT 1,11.

La qualité de l'ensilage se trouve donc affectée par le niveau de soude. Ces fortes productions d'acides gras volatils ont entraîné des pertes sensibles de matière sèche à l'étuve; nous enregistrons en effet, une différence de 3 points entre MNT et MT 1,11 .

L'équation de Schoch permet de corriger de façon satisfaisante la matière sèche (tabl. 1).

Quant au pH, il reste correct pour MNT et augmente consécutivement à la dose de soude mais reste néanmoins à un niveau relativement bas $(4,83$ et 5,47$)$. Une bonne partie de la soude a été neutralisée par les acides du milieu.

\section{Digestibilité in vitro}

Les résultats enregistrés sont rapportés dans le tableau 2 bis.

La disparition de la matière sèche passe par un maximum avec la dose 0,67 p. 100 puis diminue pour atteindre pratiquement le niveau des MNT avec la dose 1,56 p. 100. Nous avons donc choisi pour la digestibilité in vivo les doses 0,67 et 1,11 p. 100 .

\section{TABleau 2 bis}

Effet du traitement à la soude sur la digestibilité in vitro des marcs de raisin.

\begin{tabular}{c|c|c|c|c}
\hline \hline Dose de soude (\% MS) & 0 & 0,67 & 1,11 & 1,56 \\
\cline { 2 - 4 } Digestibilité ........ & 26,6 & 37,1 & 35,0 & 28,5 \\
\hline
\end{tabular}

\section{Digestibilité in vivo}

La digestibilité de tous les constituants de la ration est amélioré par le traitement $(\mathrm{P}<0,01)$. Elle passe de 10 à $25 \mathrm{p}$. 100 pour les matières azotées, de 46 à 69 p. 100 pour les matières grasses, de 32 à 48 p. 100 pour la cellulose brute et de 32 à 43 p. 100 pour la matière organique (tabl. 3).

Néanmoins, à l'exception de la digestibilité des matières grasses, la dose de 1,11 p. 100 de soude, comparativement à celle de 0,67 p. 100 , fait diminuer la digestibilité de l'azote de 7,5 points, soit 26 p. 100, et celle de la cellulose brute et de la matière organique respectivement de 12 et de 6 p. 100 sans que ces différences soient toutefois significatives. 
TABLEAU 3

Digestibilité des marcs traités ou non à la soude.

Apparent digestibility of untreated and treated grape marc.

\begin{tabular}{|c|c|c|c|}
\hline & $\begin{array}{c}\text { Marc non traité } \\
\text { Untreated grape } \\
\text { marc }\end{array}$ & $\begin{array}{l}\text { Marc traité } \\
0,67 \% \\
\text { Treated grape } \\
\text { marc } 0.67 \%\end{array}$ & $\begin{array}{l}\text { Mare traité } \\
1,11 \% \\
\text { Treated grape } \\
\text { marc } 1.11 \%\end{array}$ \\
\hline Matière sèche - Dry matter & $31,5 \pm 2,67$ & $45,4 \pm 2,97$ & $42,2 \pm 2,32$ \\
\hline Matière organique - Organic matter & $32,2 \pm 3,06$ & $44,2 \pm 2,77$ & $41,5 \pm 2,31$ \\
\hline Matières azotées - Crude protein . & $9,7 \pm 2,39$ & $28,8 \pm 5,26$ & $21,4 \pm 2,99$ \\
\hline Matière grasse - Fat $\ldots \ldots \ldots \ldots \ldots$ & $46,0 \pm 2,03$ & $70,0 \pm 4,02$ & $68,3 \pm 0,55$ \\
\hline Cellulose Weende - Cellulose Weende .. & $31,7 \pm 3,10$ & $51,5 \pm 3,72$ & $45,3 \pm 2,12$ \\
\hline Newtral detergent fibre (NDF) & $2,7,5 \pm 2,88$ & $44,5 \pm 3,67$ & $34,6 \pm 3,19$ \\
\hline Acid detergent fibre $(A D F)$ & $29,0 \pm 2,64$ & $42,2 \pm 2,97$ & $38,2 \pm 2,94$ \\
\hline Acid detergent lignin $(A D L)$ & $26,3 \pm 2,08$ & $22,7 \pm 3,17$ & $14,7 \pm 2 ; 39$ \\
\hline
\end{tabular}

Tableau 4

Valeur alimentaire des marcs de raisin traités ou non à la soude. Feeding value of untreated and treated grape marc.

\begin{tabular}{|c|c|c|c|}
\hline & $\begin{array}{l}\text { Marc non traité } \\
\text { Untreated grape } \\
\text { marc }\end{array}$ & $\begin{array}{l}\text { Marc traité } \\
\quad 0,67 \% \\
\text { Treated grape } \\
\text { marc } 0,67 \%\end{array}$ & $\begin{array}{l}\text { Marc traité } \\
\quad 1.11 \% \\
\text { Treated grape } \\
\text { marc } 1.11 \%\end{array}$ \\
\hline $\begin{array}{l}\text { MS ingérée }\left(\mathrm{g} / \mathrm{kg} \mathrm{P}^{0,75}\right) / \text { jour } \ldots \ldots \\
\text { Dry matter intake } \mathrm{g} / \mathrm{kg} W^{0.75} / \text { day }\end{array}$ & $121 \pm 12$ & $122 \pm 14$ & $129 \pm 5$ \\
\hline $\mathrm{UF} / \mathrm{kg}$ MS - FU/kg dry matter ........ & 0,13 & 0,35 & 0,33 \\
\hline UF ingérée/jour - FU intake/day $\ldots$. & 0,25 & 0,69 & 0,68 \\
\hline $\begin{array}{c}\frac{\text { UF ingérée }}{\text { UF nécessaire à entretien }} \times 100 \\
\frac{F U \text { intake }}{F U \text { for maintenance }} \times 100\end{array}$ & 55 & 153 & 151 \\
\hline $\begin{array}{l}\text { Matières azotées digestibles ingérées }(\mathrm{g}) \\
\text { Digestible nitrogen matter intake } \\
(D N M I)(g)\end{array}$ & 28 & 80 & 58 \\
\hline $\begin{array}{l}\frac{\text { MAD ingérées }}{\text { MAD nécessaire à l'entretien }} \\
\text { DNMI }\end{array}$ & 62 & 177 & 129 \\
\hline DNM for maintenance & & & \\
\hline
\end{tabular}


La digestibilité du NDF et de l'ADF augmente parallèlement à la dose de soude. En revanche, celle de la lignine diminue respectivement de 4 et de 11 points.

\section{E. Valeur alimentaire}

La quantité de matière sèche ingérée est particulièrement élevée pour les trois rations (tabl. 4). Elle est supérieure de 77 p. 100 à la moyenne des ingestibilités des fourrages verts $\left(70 \mathrm{~g} / \mathrm{kg} \mathbf{P}^{(0,75}\right)$ observée par Demarquilly \& Weiss (1970).

Le traitement à la soude triple globalement la valeur énergétique et azotée du produit qui devient alors capable d'assurer les besoins d'entretien des animaux.

\section{Discussion}

\section{A. Composition pariétale}

Sous l'effet du traitement à la soude, la teneur en NDF, en cellulose Weende et en ADF s'est accrue de façon sensible. C'est un fait assez courant comme le montrent les résultats obtenus sur les pailles de céréales par SaXena et al. (1971), Yu Yu \& EMERY (1975).

Ben-Ghedalia \& Shefet (1979) sur des sous-produits de la culture de coton observent un accroissement de 8 p. 100 pour le NDF, 21 p. 100 pour l'ADF et 32 p. 100 pour la lignine au permanganate.

Pour la paille, l'augmentation se situe entre 3 et 5 p. 100 . Il semble selon DonNely \& WEAR (1972) que ce phénomène soit lié à la composition chimique des produits étudiés, notamment à leur richesse en protéines insolubles et en corps phénoliques, ce qui est le cas des marcs de raisin. Il se produirait au cours des processus d'analyse des complexes de types phlobaphênes, insolubles dans le solvant NDF (DonNELY \& Wear, 1972). Par ailleurs, le traitement à la soude en solubilisant une partie de la lignine (REXen \& Thomsen, 1976) favorise le contact de cette dernière avec les protéines; il se formerait alors selon VAN SoEST (1967) des substances polymérisées brunes de haut poids moléculaire appelées souvent «artefact lignin». Ces derniers affecteraient la détermination de la lignine, de la cellulose et à moindre degré, celle des sucres simples et des hémicelluloses en gonflant artificiellement leur évaluation.

Ces réactions, seraient particulièrement favorisées par le passage à l'étuve à une température supérieure à $50^{\circ}$ et par un pH basique (Ellis, Matrone \& MaYNARD, 1946) ce qui est souvent le cas pour les produits traités à la soude.

Seule la fraction lignine, plus cutine, voit sa teneur diminuée. La forte diminution observée (29 p. 100 en moyenne) est probablement liée à une solubilisation de la cutine. En effet, nous constatons en utilisant la méthode permanganate de VAN SoesT que la teneur en cutine des marcs de raisin se situe à 26,14 et 7 p. 100 respectivement pour MNT, MT 0,67, MT 1,11.

Dans cet ordre d'idée, une partie de la cutine solubilisée serait évacuée par le jus d'ensilage; il en résulterait alors une autre cause d'augmentation de la teneur du produit en NDF et en ADF. 


\section{B. Facies fermentaire et quantités ingérées}

Le traitement à la soude entraîne une production importante d'acide acétique et d'acide butyrique et une décroissance de la production d'acide lactique. Cette dernière observation s'explique probablement par sa réutilisation par les bactéries butyriques (Sauvant \& Gouet, 1979) ce qui expliquerait les teneurs élevées en acide butyrique.

Des observations similaires sont faites par Shultz, Ralston \& Shultz (1974) sur un ensilage de paille de céréale préalablement traité à la soude. Toutefois, les valeurs que nous rapportons pour l'acide butyrique restent particulièrement élevées ( $44 \mathrm{~g} / \mathrm{kg} \mathrm{MS}$ ) ; la méthode de dosage que nous avons utilisée est probablement en cause car elle est moins spécifique que le dosage effectué par chromotographie en phase gazeuse.

De cette dégradation des paramètres fermentaires des ensilages, nous pouvions nous attendre à une diminution de l'ingestibilité.

Mais, il n’est pas constaté de différence significative de consommation entre les trois types de marc. Ce résultat n'est pas pour autant étonnant puisqu'il est admis que la diminution de consommation est essentiellement liées à une forte acidité du produit, ce qui n'est pas le cas des marcs traités, et à la putréfaction des protéines qui développe dans les ensilages des odeurs nauséabondes. Cette putréfaction est limitée dans le cas des mares de raisin dont l'azote est très peu fermentescible (moins de 2 p. 100 par rapport à l'azote total).

Le traitement à la soude, n'augmente pratiquement pas l'ingestibilité du produit comme cela est rapporté pour la paille traitée (BIEN-AImÉ, 1979); elle s'est accrue seulement de 6 p. 100 pour MT, 1,11 par rapport à MNT (121 contre $\left.129 \mathrm{~g} / \mathrm{kg} \mathrm{P}^{0,75}\right)$. En moyenne, l'ingestibilité des marcs de raisin est 3,5 à 4 fois plus élevée que celle enregistrée respectivement pour la paille normale et la paille traitée à 4 p. 100 de soude (BIEN-AIMÉ, 1979).

Ce niveau élevé de consommation malgré la faible digestibilité du produit est peut être lié à une vitesse de transit accéléré, compte tenu de la taille des particules qui varie entre 5 et $15 \mathrm{~mm}$ (Alwash \& Thomas, 1974).

\section{Digestibilité et valeur alimentaire des rations}

Après traitement, la digestibilité des marcs de raisin s'est accrue de 12 points pour MT 0,67 p. $100(\mathrm{P}<0,01)$ et de 9 points pour MT 1,11 ( $<<0,01)$.

La soude doit agir particulièrement sur la digestibilité de l'azote puisque la digestibilité de celui-ci passe de 10 à 29 et à 21 p. $100(\mathrm{P}<0,01)$ respectivement pour MNT, MT 0,67 et MT 1,11. Cette plus grande labilité de l'azote a probablement permis une amélioration de la digestibilité de l'ensemble des constituants. Une action spécifique sur la matière grasse doit être signalée : en effet, son utilisation digestive augmente en moyenne de 23 points $(\mathrm{P}<0,01)$.

La diminution de digestibilité de la matière sèche observée pour MT 1,11 par rapport au MT 0,67 (6 p. 100) confirme nos résultats in vitro. Elle ne semble pas s'expliquer par la quantité de soude ingérée, compte tenu du fait qu'elle se situe à $22 \mathrm{~g}$ alors que des moutons consommant de la paille traitée à 4 p. 100 de soude peuvent en ingérer $35 \mathrm{~g}$ par jour sans que la digestibilité de la ration en soit affectée (Revue de 
JACKSON, 1977). Cependant, après traitement à la soude, la formation de substances toxiques du type quinonique par oxydation des corps phénoliques est une hypothèse probable.

La digestibilité du NDF, de ADF et de la cellulose brute s'est améliorée sous l'action de la soude. Selon BACON (1979), la soude agit en coupant les liaisons hydrogènes des composants pariétaux et fait disparaître une bonne partie des composés phénoliques. De même, elle déplacerait des groupements acétyles qui sembleraient être des dépresseurs de la digestibilité en cachant les sites de digestion.

La digestibilité de la cellulose Weende (43 p. 100 en moyenne) est plus élevée $(\mathrm{P}<0,01)$ que celle de l'ADF et de NDF (respectivement 36 et $35 \mathrm{p} .100$ ). Ce résultat n'est pas étonnant compte tenu que l'ADF et le NDF renferment la totalité de la lignine, principe très peu digestible. Mais, il est également connu (VAN SoEst, 1963) que la valeur de l'ADF et de NDF augmente avec le temps de séjour des échantillons à l'étuve ; ce temps est habituellement plus long pour les fèces. La digestibilité de ces deux fractions se trouve alors artificiellement diminuée.

Nous observons par ailleurs une diminution régulière de la digestibilité de l'ensemble lignine-cutine, parallèlement à la dose de soude, mais ceci semble normal compte tenu que sa teneur diminue sous laction du traitement.

L'amélioration de la digestibilité de la matière organique permet d'augmenter dans de fortes proportions la valeur énergétique des mares traités. Considérant que la quantité ingérée ne diminue pas, la quantité d'UFL ingérée passe de 0,20 à 0,70 , soit un niveau d'alimentation de 50 p. 100 supérieur à celui de l'entretien alors que les mares non traités n'assurent que 50 p. 100 des mêmes besoins d'entretien de moutons pesant environ $50 \mathrm{~kg}$.

En se référant aux résultats présentés par Hoden (1973), JACKson (1977), HarTON (1978) et BIEN-AIMÉ (1979), les mares de raisin ont une valeur alimentaire supérieure à celle de la paille traitée à la soude ou complémentée avec un concentré.

\section{Conclusion}

Les marcs de raisin distribués comme seuls composants de la ration, malgré une ingestibilité de $124 \mathrm{~g} / \mathrm{kg} \mathbf{P}^{0,75}$ n'assurent pas les besoins dentretien de moutons de $50 \mathrm{~kg}$ qui perdent du poids; il est nécessaire de les complémenter avec un concentré riche en azote. Cette nécessité n'est pas recommandable d'un point de vue économique dans les pays maghrébins producteurs de marcs.

Le traitement à la soude, en modifiant la structure des glucides pariétaux et surtout en rendant plus disponible l'azote, améliore de façon importante la digestibilité de l'ensemble des constituants de la matière organique. De ce fait, la valeur énergétique du produit passe de $0,13 \mathrm{UFL} / \mathrm{kg}$ MS à $0,34 \mathrm{UFL}$ en moyenne. La quantité ingérée n'étant pas modifiéc, la valeur alimentaire du produit s'établit alors à 150 p. 100 des besoins d'entretien.

Les marcs de raisin traités à la soude constituent donc un aliment particulièrement intéressant pour des moutons à l'entretien ou à faible croissance.

Acception pour publication en avril 1983. 


\section{Summary \\ Feeding value of grape marc \\ II - Effect of sodium hydroxide treatment on the feeding value of steam extracted grape marc silage in sheep}

Digestibility of grape marc (given ad libitum), treated or not with 0.67 p. 100 or 1.11 p. 100 sodium hydroxide $(\mathrm{NaOH})$ relative to dry matter was studied in groups of 4 sheep.

Sodium hydroxide treatment increased the digestibility from 32 p. 100 (non treated marcs) to 44 p. 100 (marcs treated with 0.67 p. $100 \mathrm{NaOH}$ ). Treatment with 1.11 p. 100 $\mathrm{NaOH}$ led to a slight decrease in the organic matter digestibility (41 p. 100). The improvement of digestibility concerned all the marc components and particularly cellulose Van Soest and lipids whose digestibility increased from 25 to 61 p. 100 and from 46 to 69 p. 100 , respectively. Nitrogen digestibility remained low on an average $(20$ p. 100), but varied from 10 (non treated marc) to 29 p. 100 (marc treated with 0.67 p. $100 \mathrm{NaOH}$ ).

Voluntary feed intake was particularly high $(124 \mathrm{~g})$ on an average $\left./ \mathrm{kg} \mathrm{W}^{0.5 \pi}\right)$. It was not modified by the $\mathrm{NaOH}$ treatment despite a reduction in the silage quality (butyric acid increased from $1.4 \mathrm{~g}$ to $44 \mathrm{~g} / \mathrm{kg}$ dry matter when the addition of sodium hydroxide increased from 0 to 1.11 p. 100).

Because of the high voluntary intake together with the improvement of organic matter digestibility, the feeding value of treated grape marc met 1.5 fold the requirements for maintenance while that of non treated grape marc met only 55 p. 100 of the requirements.

Accordingly, $\mathrm{NaOH}$ treated grape marc is a valuable feed for animals in maintenance or with a low production.

\section{Références bibliographiques}

Alwash A.H., Thomas P.C., 1974. Effect of size of hay particle on digestion in the sheep. J. Sci. Fed. Agric., 25, 139-147.

BACON J.S.D., 1979. Plant cell-wall digestibility and chemical structure. Rowett Ann. Rep. Res. Inst., 35, 99-108.

Ben-Ghedalia D., Shefet S., 1979. The effect of sodium hydroxyde and ozone treatments on the composition and in vitro organic matter digestibility of cotton straw. Nut. Rep. int., 20 (2), 179-182.

Bien-Aimé A., 1979. Facteurs de variation de la digestibilité des pailles de céréales. Thèse de Doctorat de $3^{c}$ cycle, Université de Montpellier, $118 \mathrm{p}$.

Demarquilly C., Weiss Ph., 1970. Tableaux de la valeur alimentaire des fourrages. S.E.I., I.N.R.A., route de Saint-Cyr, 78000 Versailles.

Donnely E., Wear J., 1972. Acid detergent method for reduction of tanin interference in determining lignin of sericea lespedeza. Agr. J. Madison, 64 (6), 838-839.

Dumont R., Tisserand J.L., 1978. Valeur alimentaire d'un marc de raisin déshydraté. Ann. Zootech., 27, 631-637.

Economides S., Hadjidemetriou, 1974. The nutritive value of some agricultural by-products (communication personnelle).

Ellis G.H., Matrone G., Maynard L.A., 1946. A 72 p. $100 \mathrm{H}_{2} \mathrm{SO}_{4}$ method for the determination of lignin and its use in animal nutrition studies. J. Anim. Sci., 5, 285-295.

Hammouda F., 1980. Action de trois traitements employés à différentes doses sur la composition chimique et la digestibilité in vitro des marcs de raisin épuisés et ensilés. Thèse d'ingéniorat, I.N.A., El-Harrach, Algérie. 
Hammouda F., LaRwence A., 1981. Intérêt des marcs de raisin dans l'alimentation des ruminants. IV $V^{e s}$ Journées d'études des productions animales, I.N.A., El-Harrach, Algérie.

Hoden A., 1973. Valeur alimentaire des fourrages pauvres pour les ruminants. Effet de la complémentation azotée. D.E.A., Université de Clermont-Ferrand.

Harton G.M.S., 1978. The intake and digestibility of ammoniated cereal straw by cattle. Can. J. Anim. Sci., 58, 471-478.

I.N.R.A., 1978. Alimentation des ruminants. Ed. I.N.R.A., route de Saint-Cyr, 78000 Versailles, $597 \mathrm{pp}$.

JACKSON S., 1977. The alkali treatment of straws. Anim. Feed. Sci. Technol., 2, 105-130.

LarWENCE A., Yahioui A., 1983. Influence de 8 sources azotées de complémentation sur l'utilisation digestive par le mouton de marc de raisin épuisé et ensilé. Ann. Zootech., 32, $103-116$.

Mazziotti D.I., Celso P., Rizzo V., Bernardini G., 1970. Digestibilita e valore nutritivo di farina di extrazione di vinaccioli diverso contenuto di filtra grezza. Annali dell istituto sperimentale per la zootechnica, 3 (1), 71-77.

Reyne Y., Garambois X., 1977. Valeur alimentaire chez le mouton de l'ensilage de marc de raisin épuisé. Ann. Zootech., 26, 471-479.

ReXeN F.P., THOMSEN K.V., 1976. The effect on digestibility of a new technique for alkali treatment of straw. Anim. Feed. Sci. Technol., 1, 73-83.

Sauvant D., Gouet P., 1979. Les relations entre les processus fermentaires : conséquences pour l'appréciation quantitative de la qualité de la conservation. In : La Conservation par ensilage. C.A.A.A., I.N.A., Paris-Grignon, pp. $41-77$.

Saxena S.K., Otterby D.E., Donker J.D., Good A.L., 1971. Effects of feeding alkalitreated oat straw supplemented with soybean meal or nonprotein nitrogen on growth of lambs and on certain blood and rumen liquor parameters. J. Anim. Sci., 33, 485-490.

Scносн W., 1949. Die bei der Trocknung von Selageproben im Trockenschrank auftretender Verluste von flüchtigen Saüren und Basen. Mitteilungen aus dem Gebiete der Lebensmitteluntersuchung und Hygiene, 40, 179-189.

Shultz T.A., Ralston A.T., Shultz E., Effect of various additives on nutritive value of Ryegrass straw silage. I - Laboratory silo and in vitro dry matter digestion observations. J. Anim. Sci., 39, 920-924.

TIlley J.M.A., Terry R.A., 1963. A two stage technique for the in vitro digestion of forage crops. J. Brit. Grassld. Soc., 18, 104-111.

Van Soest P.J., 1963. New chemical procedures for evaluating forage. 55th annual meeting of the american society of animal science. August 13.

VAN SoEst P.J., 1967. Use of detergents in the analysis of fibrous feeds. II A - Rapid method for the determination of fiber and lignin. J.A.O.A.C., 46 (5), 829-835.

YU YU J.W.T., EMERY R.S., 1975. Estimated value of treated forages for ruminants. J. Anim. Sci., 41, 1742-1751. 